\title{
A successful pregnancy of a dialysis patient in the Eastern Caribbean
}

\author{
E Mohammed ${ }^{1}$, A Ramrattan ${ }^{2 *}$, A Sahadeo ${ }^{3}$, E Brathwaite $^{3}$ and S Soobrattie ${ }^{3}$ \\ ${ }^{1}$ Department of Internal Medicine, Northwest Regional Health Authority, Port-of-Spain General Hospital and University of the West Indies, St Augustine, \\ Trinidad and Tobago \\ ${ }^{2}$ Department of Internal Medicine, Northwest Regional Health Authority, Port-of-Spain General Hospital, Trinidad and Tobago \\ ${ }^{3}$ Department of Obstetrics and Gynecology, Northwest Regional Health Authority, Port-of-Spain General Hospital, Trinidad and Tobago
}

\begin{abstract}
Pregnancy is rarely seen in women with End Stage Renal Disease (ESRD) due to decreased fertility and in the event of conception, the inability of the stable dialysis patient to adapt to the physiological demands of pregnancy. Despite the increasing incidence of pregnancy in dialysis patients in developed countries, the Eastern Caribbean has only now had its first case and here we have an in-depth look at what is essential to have a successful outcome for both mother and fetus.
\end{abstract}

\section{Introduction}

Since the first internationally recognized case of a pregnant woman on dialysis in 1971 by Confortini, et al. [1], outcomes of successful pregnancies have significantly improved over the years and is largely attributed to improvements made with dialysis delivery and a multidisciplinary team management of these patients. The following illustrates the successful pregnancy in a dialysis patient in the Twin Republic of Trinidad and Tobago at the Port-of-Spain General Hospital (POSGH). To date, there are no known cases of a successful pregnancy in a dialysis patient in the Caribbean.

\section{Case report}

This 33-year-old female with ESRD secondary to primary glomerulonephritis on hemodialysis (HD) for over 12 months, presented to the Nephrology team with a positive home pregnancy test. She was referred to the Obstetrics team at the POSGH for antenatal care with a previous history of 5 first trimester miscarriages.

Medications at conception: Renvela $800 \mathrm{mg}$ po tds, Furosemide 40 $\mathrm{mg}$ po bd, Folic acid $5 \mathrm{mg}$ po od, Venofer $100 \mathrm{mg}$ IV every 2 weeks and Mircera 100 mcg SC monthly.

Medications were immediately changed to: Folic Acid $5 \mathrm{mg}$ po od, Venofer $100 \mathrm{mg}$ IV every 2 weeks, Recormon 4000 U SC three times weekly, Caltrate/Vit D3 600mg/400 IU 2 tablets daily, and IFA 2 teaspoon daily.

A pelvic ultrasound scan (USS) confirmed a single live intrauterine pregnancy at 8 weeks 3 days, with an estimated due date (EDD) of the $15^{\text {th }}$ March 2019 and a dating scan done at 12 weeks and 6 days, with crown rump length of $64.35 \mathrm{~mm}$ confirming this.

The patient's dialysis was increased to 6 times weekly, 3 at POSGH and 3 at John Hayes Memorial Kidney Foundation with the following protocols: HD 3 and $1 / 2$ to 4 hours each session, dialysate flow $500 \mathrm{ml} /$ min, Use of a $170 \mathrm{H}$ biocompatible dialyzer membrane, Heparin $500 \mathrm{U}$ bolus and $500 \mathrm{U}$ maintenance every hour, low pump speed at 250-300 $\mathrm{ml} / \mathrm{min}$, Bicarbonate adjusted to $25 \mathrm{meq} / \mathrm{L}$, increased target weight of $0.5 \mathrm{~kg}$ every 2 weeks from second trimester and one on one nursing.

Attempts to change the dialysate to a higher potassium of 3-3.5 $\mathrm{mmol} / \mathrm{L}$ was unsuccessful. She was therefore allowed a normal potassium intake and referred to a dietician to assist in increasing caloric requirements to $2500-3000 \mathrm{kcal} /$ day and protein intake up to $1.5-1.8 \mathrm{~g} / \mathrm{kg} /$ day.

Aspirin $81 \mathrm{mg}$ po od was commenced from 12 weeks gestation to reduce the risk of pre-eclampsia and the patient was followed up weekly by the Obstetrics team and Nephrology teams and then twice weekly by the Obstetrics team from 26 weeks gestation. Routine bloods done showed the patient was blood group A positive, HIV, Hepatitis B, Hepatitis $C$ and VDRL negative.

An echocardiogram done at 30 weeks gestation to assess cardiac function showed a normal ejection fraction with trivial mitral and tricuspid regurgitation.

Ferritin levels ranged from $84-132.3 \mathrm{ng} / \mathrm{ml}$ and was done every 6 weeks from the second trimester. Folate and B12 levels done at the start of third trimester was $12.38 \mathrm{ng} / \mathrm{ml}$ and $211.9 \mathrm{pg} / \mathrm{ml}$ respectively, both within normal limits. The patient had liver function tests done routinely every week in the third trimester all of which were within normal limits.

Then patient's pre-pregnancy Hemoglobin was $8 \mathrm{~g} / \mathrm{L}$ and the aim was to maintain this during pregnancy. Recormon dosage was

${ }^{\star}$ Correspondence to: Amit Ramrattan, Department of Internal Medicine, Northwest Regional Health Authority, Port-of-Spain General Hospital, Trinidad and Tobago, E-mail: amitramrattan400@gmail.com

Key words: pregnancy, end stage renal disease, dialysis

Received: March 03, 2021; Accepted: March 17, 2021; Published: March 22, 2021 
decreased to twice weekly at the start of her third trimester and then once weekly at 30 weeks gestation.

As part of her antenatal care, the patient had a $75 \mathrm{~g}$ oral glucose tolerance test (OGTT) done at 16- and 26-weeks' gestation which were normal. Additionally, daily home blood pressure (BP) monitoring was performed which revealed blood pressures within normal limits (the diastolic BP remained below $80 \mathrm{mmhg}$ for the entire pregnancy). Delivery was aimed for 33 to 34 weeks gestation after discussion at a multidisciplinary team meeting involving Obstetrics, Nephrology, Neonatology and Anesthetics and she completed Dexamethasone prior to 34 weeks gestation. However, at 34 weeks gestation, the patient was noted to have an elevated white cell count and was started on prophylactic oral cefuroxime for a suspected urinary tract infection while awaiting the results of a midstream urine. At this gestation, she had 2 episodes of an elevated BP in antenatal clinic 153/77 and was admitted for BP monitoring but antihypertensives were not required.

Induction of labour scheduled at $33+5$ gestation was delayed due to unavailable Neonatal ICU facilities at that time. At $34+4$ gestation, the patient had preterm prelabour rupture of membranes (P-PROM) and was started on oral clindamycin to be switched to Benzyl Penicillin once in active labour. The patient went into spontaneous precipitate labour a few hours after P-PROM and delivered a live preterm male infant with APGAR scores of 9 at one minute and of 9 at 5 minutes. The mid-stream urine report obtained immediately post-delivery showed Acinetobacter and Group B Streptococcus resistant to cefuroxime and sensitive to norfloxacin. She was therefore started on oral norfloxacin. In her immediate postpartum period, her hemodialysis sessions were reduced to three times weekly and she had heparin free dialysis due to breastfeeding. The patient's infant is doing well currently. She was counselled on contraception and avoiding pregnancy until 2 years post renal transplant and had a Levonorgestrel intrauterine device placed 2 months post-delivery (Tables 1-3).

\section{Discussion}

Data on the epidemiology of pregnancy in dialysis worldwide is limited with literature offering many case series, surveys and literature

Table 1. Complete blood count trend

\begin{tabular}{|c|c|c|c|c|}
\hline POG & WBC (10 $/ \mathbf{L})$ & Hb (g/L) & MCV (fL) & Platelet (10 $/ \mathbf{L})$ \\
\hline $11+0$ & 14.5 & 9.0 & 95.1 & 173 \\
\hline $15+3$ & 10.9 & 7.5 & 99.4 & 227 \\
\hline $16+3$ & 12.2 & 8.1 & 98.8 & 255 \\
\hline $17+5$ & 8.3 & 7.4 & 100.2 & 207 \\
\hline $18+3$ & 10.1 & 7.5 & 99.9 & 216 \\
\hline $19+3$ & 7 & 7.9 & 100 & 210 \\
\hline $20+4$ & 11.6 & 8.3 & 101.2 & 223 \\
\hline $22+3$ & 11.9 & 8.2 & 99.2 & 254 \\
\hline $23+4$ & 11.7 & 7.6 & 104.3 & 258 \\
\hline $24+3$ & 11.3 & 7.6 & 98.1 & 272 \\
\hline $25+4$ & 11.3 & 7.9 & 97.3 & 284 \\
\hline $26+3$ & 10 & 8 & 96.9 & 267 \\
\hline $27+3$ & 10.1 & 7.4 & 95.5 & 245 \\
\hline $28+3$ & 8.9 & 7.6 & 95.9 & 237 \\
\hline $29+3$ & 11.4 & 6.9 & 97.0 & 255 \\
\hline $30+0$ & 10.5 & 8 & 96.5 & 219 \\
\hline $32+5$ & 9.1 & 6.7 & 96.7 & 160 \\
\hline $33+3$ & 26.9 & 7.4 & 96.1 & 303 \\
\hline $34+4$ & 15.5 & 8.1 & 95.3 & 243 \\
\hline
\end{tabular}

POG: Period of gestation; WBC: White blood cell; Hb: Hemoglobin; MCV: Mean corpuscular volume.
Table 2. Biochemistry profile trend

\begin{tabular}{|c|c|c|c|c|c|}
\hline POG & $\mathbf{K}(\mathbf{m m o l} / \mathbf{L})$ & $\mathbf{B U N}(\mathbf{m g} / \mathbf{d l})$ & $\begin{array}{c}\mathbf{c r e a t e}(\mathbf{m g} / \\
\mathbf{d} \mathbf{)}\end{array}$ & $\mathbf{C a}(\mathbf{m g} / \mathbf{d l})$ & $\mathbf{U A ~ ( m g / d l )}$ \\
\hline $15+3$ & 3.7 & 32 & 7.5 & 8.1 & - \\
\hline $16+3$ & 3.6 & 39 & 7.96 & 8.7 & - \\
\hline $17+5$ & 3.4 & 23 & 7.8 & 8.1 & - \\
\hline $18+3$ & 4.1 & 27 & 7.75 & 8.4 & - \\
\hline $19+3$ & 3.7 & 28 & 7.36 & 8.2 & 5.7 \\
\hline $20+4$ & 4.8 & 30 & 7.35 & 8.6 & - \\
\hline $23+4$ & 3.5 & 28 & 8.3 & 8.8 & 5.3 \\
\hline $24+3$ & 3.0 & 30 & 8.35 & 9.1 & 6.4 \\
\hline $25+4$ & 3.7 & 33 & 8.38 & 9.4 & 6.3 \\
\hline $26+3$ & 3.7 & 41 & 10.19 & 8.9 & 7.4 \\
\hline $27+3$ & 3.8 & 35 & 7.62 & 7.2 & - \\
\hline $28+3$ & 3.7 & 36 & 9.49 & 8.5 & 6.9 \\
\hline $29+3$ & 3.8 & 51 & 10.01 & 8.2 & - \\
\hline $30+0$ & 3.5 & 16 & 5.29 & 8.5 & - \\
\hline $31+4$ & 3.9 & 25 & 7.39 & 8.3 & 5.1 \\
\hline $32+5$ & 3.7 & 37 & 9.85 & - & - \\
\hline $33+3$ & 3.4 & 45 & 9.31 & 9.3 & 7.5 \\
\hline
\end{tabular}

POG: Period of gestation; K: Potassium; BUN: Blood urea nitrogen; Creat: Creatinine; Ca: Calcium; UA: Uric acid.

Table 3. Pelvic and obstetric ultrasound scans

\begin{tabular}{|c|c|c|c|c|}
\hline POG & EFW/g & $\begin{array}{c}\text { Umbilical } \\
\text { Artery } \\
\text { Doppler RI }\end{array}$ & $\begin{array}{c}\text { Liquor } \\
\text { Volume }\end{array}$ & Comments \\
\hline $12+6$ & - & - & - & EDD -15/03/19 \\
\hline $21+0$ & 432 & - & normal & normal anomaly scan \\
\hline $24+0$ & 706 & 0.72 & normal & normal growth scan \\
\hline $26+0$ & 1009 & 0.73 & normal & normal growth scan \\
\hline $28+0$ & 1275 & 0.62 & normal & normal growth scan \\
\hline $30+0$ & 1692 & 0.66 & normal & normal growth scan \\
\hline $32+0$ & 1690 & 0.70 & normal & normal growth scan \\
\hline
\end{tabular}

POG: Period of gestation; EFW: Estimated fetal weight.

reviews. What is recognized throughout the literature is the improving fetal outcomes of dialysis patients who became pregnant [2]. Women with chronic kidney disease (CKD) and especially those on dialysis tend to have diminished fertility attributed to menstrual irregularities, dysfunction of the hypothalamic-pituitary-gonadal axis which affects follicular stimulating and luteinizing hormone and subsequent estrogen and progesterone, hyperprolactinemia and causes anovulatory states and in some cases early menopause. At the Port of Spain General Hospital, this was the first case of a dialysis patient becoming pregnant and this was confirmed via pelvic ultrasound as higher rates of false positive home pregnancy test from falsely elevated beta-subunit of human chorionic gonadotropin are seen in CKD.

Pregnancy and dialysis carry risks to both mother and fetus. Maternal complications include a higher risk of miscarriage, premature rupture of membranes, pregnancy induced hypertension and preeclampsia, maternal hypotension in dialysis, worsening anemia in pregnancy and a higher risk of antepartum hemorrhage due to heparin on dialysis. Fetal complications include intrauterine growth restriction (IUGR), polyhydramnios and preterm labor. In a review of case series by the Clinical Kidney journal, pre-term births occur in $83 \%$ of live births with an average gestational age of 32 weeks or less and growth retardation complications of $28-36 \%$ and malformations in $10 \%$ of cases [3].

The consensus on literature review of an optimal dialysis prescription suggest that more than 20 hours a week cumulative 
dialysis improved live births [3-5],where increased frequency and length of dialysis improved infant survival and decreased prematurity [3-5]. This also allows for a better volume control of the patient as well as electrolytes. A pre-dialysis BUN of less than $50 \mathrm{mg} / \mathrm{dl}$ was aimed for as a lower BUN correlated to better birthweight and less IUGR and polyhydramnios and preterm labour [5]. Our patient's dialysis prescription was therefore tailored to meet the above parameters.

Ideally, the dialysate in this patient should have been changed to a higher potassium of 3-3.5 mmol/L but allowing a normal potassium diet compensated for this. Hypokalemia normally should be avoided as it can cause cellular shifts of ions which can potentially affect blood pressures. Having frequent dialysis can cause an alkalemia which can affect oxygen delivery to tissues and fetus, and this was avoided by changing the bicarbonate concentration to $25 \mathrm{meq} / \mathrm{L}$. Heparin dose was halved as mentioned earlier as a balance in attempt to avoid antepartum hemorrhage but also avoid clotting in dialysis which can worsen anemia $[6,7]$.

Pre-eclampsia is a major maternal complication in patients on dialysis which can cause IUGR and fetal demise and this patient was followed up very closely. At 12 weeks gestation low dose Aspirin was prescribed to reduce the risk of pre-eclampsia and she recorded home blood pressure logs which were reviewed weekly. Given that Erythropoiesis stimulating agents (ESA) can cause hypertension and also the relative dilutional anemia in pregnancy, the aim was to keep her Hemoglobin $(\mathrm{Hb})$ close to that of her pre-pregnancy range, a relative balance to avoid overuse of her ESA and trigger a pre-eclamptic episode or a thrombotic complication given the additive risk of ESA's and pregnancy both potentiating a pro-thrombotic environment. Her folate, B12 and ferritin levels were monitored in pregnancy along with her Hb levels.

To keep up with the growing needs of the fetus, placenta and natural weight gain but also to avoid volume overload from her CKD, a weight gain of $0.5 \mathrm{~kg}$ every 2 weeks was allowed with regular clinical review and further adjustments made to target weight if necessary. This was an essential clinical component of avoiding hypertension related complications. A normal diet was allowed which was made possible by having frequent dialysis, and the patient was referred to a dietician to for a protein intake of $1.5-1.8 \mathrm{~kg} /$ day. Having a normal diet avoided hypophosphatemia that comes with intensive dialysis which is needed for fetal growth. Hypocalcemia should also be avoided as it can impair fetal skeletal growth and our patient was placed on Caltrate/Vit D3.

This patient was delivered at 34 weeks gestation after consensus with the multi-disciplinary teams comprising the Nephrologists, Obstetricians, Neonatologists and Anesthetists. The decision to deliver at 34 weeks was based on the premise that prolonging the pregnancy carried a higher risk of the maternal complications as mentioned prior and a possible increased chance of fetal demise. This time period was deemed a good balance for fetal maturity and avoiding further complications. Elective Caesarian section delivery is the preferred mode of delivery in dialysis patients and was being considered in our patient. As part of her preparation for delivery, dexamethasone was given for fetal lung maturity. However, our patient had a spontaneous rupture of her membranes which led to an uncomplicated spontaneous vaginal delivery.

There were several patient related factors that may have contributed to the successful pregnancy. This includes the fact that the patient had a primary glomerulonephritis. Diabetes Mellitus, hypertension or a systemic disease may increase the risk developing pre-eclampsia and vasculopathy, causing IUGR and fetal demise. Whether these comorbidities affect pregnancy outcomes is unclear from the literature. This patient also had a significant residual renal function which may have contributed to better fluid control and therefore BP control. Patient compliance is likely to have also been a contributing factor to this successful outcome.

\section{Conclusion}

In conclusion, pregnancy and dialysis requires a multidisciplinary approach comprising Nephrology, Obstetrics, Neonatology and the dialysis nursing staff, as well as close monitoring of several parameters including dialysis dose, anemia management, calcium and phosphorus balance, blood pressure control with optimal fluid balance and target weights throughout the pregnancy, adequate nutrition and fetal growth monitoring including umbilical artery dopplers. Women on long term dialysis should be offered a chance to conceive once counselled on the complications and willing to commit to the intensive regime that it entails to have a chance of a successful pregnancy. Even with the limited dialysis resources available in the Eastern Caribbean, this case has highlighted that with commitment and a concerted effort from the multidisciplinary team and patient can result in a successful pregnancy. The management of this patient also adds to the limited body of evidence on optimal management to achieve a successful pregnancy.

\section{References}

1. Confortini P, Galanti G, Ancona G (1971) Full-term pregnancy and successful delivery in a patient on chronic haemodialysis. Proc Eur Dial Transplant Assoc 8: 74-80.

2. Hladunewich MA, Hou S, Odutayo A, Cornelis T, Pierratos A, et al. (2014) Intensive hemodialysis associates with improved pregnancy outcomes: A Canadian and United States cohort comparison. J Am Soc Nephrol 25: 1103-1109. [Crossref]

3. Manisco G, Potì M, Maggiulli G, Di Tullio M, Losappio V, et al. (2015) Pregnancy in end-stage renal disease patients on dialysis: How to achieve a successful delivery. Clin Kidney J 8: 293-299. [Crossref]

4. Tangren J, Nadel M, Hladunewich MA (2018) Pregnancy and end-stage renal disease. Blood Purif 45: 194-200. [Crossref]

5. Sachdeva M, Barta V, Thakkar J, Sakhiya V, Miller I (2017) Pregnancy outcomes in women on hemodialysis: a national survey. Clin Kidney J 10: 276-281. [Crossref]

6. Al-Saran KA, Sabry AA (2008) Pregnancy in dialysis patients: a case series. J Med Case Rep 2: 10.

7. Piccoli GB, Conijn A, Consiglio V, Vasario E, Attini R, et al. (2010) Pregnancy in dialysis patients: Is the evidence strong enough to lead us to change our counseling policy? Clin J Am Soc Nephrol 5: 62-71. [Crossref]

Copyright: (C2021 Mohammed E. This is an open-access article distributed under the terms of the Creative Commons Attribution License, which permits unrestricted use, distribution, and reproduction in any medium, provided the original author and source are credited. 